> Les récepteurs couplés aux protéines G (RCPG), aussi appelés protéines à sept domaines transmembranaires (7TM), représentent la plus grande famille de protéines. Elle comprend, chez l'homme, environ 900 membres. Ces protéines se lient à une grande variété de ligands ce qui entraîne l'activation de voies de signalisation impliquées dans divers processus cellulaires. Certaines protéines à 7TM, communément appelés orphelines, n'ont pas de ligand identifié, mais semblent jouer un rôle important dans la modulation de la fonction cellulaire via leurs activités constitutives ou leurs interactions avec d'autres protéines à 7TM. Certains virus synthétisent des protéines orphelines à 7TM homologues aux récepteurs de chimiokines humains pour détourner les fonctions de la cellule hôte et promouvoir leur réplication et leur dissémination. En effet, les protéines virales à 7TM sont capables de former des homomères ou des hétéromères avec d'autres protéines virales à 7TM, voire avec des protéines à 7TM de la cellule hôte. L'hétéromérisation des protéines virales à 7TM constitue une stratégie pertinente pour contrôler les fonctions de la cellule hôte. <

Les protéines membranaires présentent un intérêt particulier parce qu'elles se trouvent à l'interface entre les milieux extraet intracellulaires. La plupart des protéines membranaires codées par le génome humain sont, soit des récepteurs, soit des transporteurs ou des enzymes. Certains de ces récepteurs font partie de la famille des récepteurs couplés aux protéines G (RCPG) qui compte environ 900 membres chez l'homme [1]. Leur dénomination de récepteur implique l'activation de ces protéines par un ligand. De fait, les RCPG sont ciblés par un grand nombre de ligands incluant des photons, des ions, des métabolites, des lipides et des peptides. La résolution récente de la structure de plusieurs RCPG a confirmé la grande conservation structurale des différents membres de cette famille [2] $(\rightarrow)[25,26]$. $(\rightarrow)$ Voir les articles de G. Lebon et C. Tate (page 876), et S. Granier, page 870 de ce numéro

\section{Oligomérisation des protéines humaines et virales à sept domaines transmembranaires}

Nouvelle stratégie virale pour manipuler la cellule hôte

\section{Ralf Jockers, Florence Gbahou, Kenjiro Tadagaki, Maud Kamal}

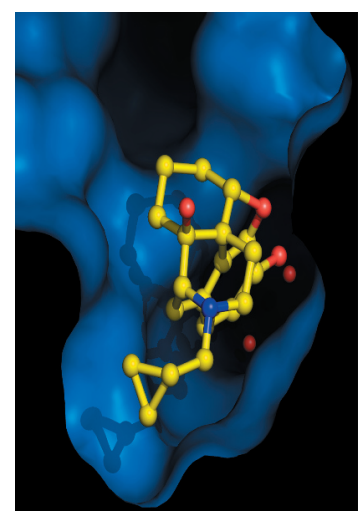

Inserm U1016, Institut Cochin, Paris, France ; CNRS UMR 8104, Paris, France ; Université Paris Descartes, Sorbonne Paris-Cité, Paris, France.

ralf.jockers@inserm.fr

Ces RCPG se composent de sept hélices transmembranaires reliées par trois boucles intra- et extracellulaires, une extrémité amino-terminale localisée du côté extracellulaire et une extrémité carboxy-terminale localisée du côté intracellulaire. À cause de cette structure commune, ils sont également dénommés protéines à sept domaines transmembranaires (7TM). Des études récentes de comparaison des génomes entièrement séquencés d'organismes très divers suggèrent une origine commune de la plupart, voire de la totalité des protéines à 7TM, avec l'apparition des premiers membres chez les métazoaires. [3].

Hormis leur rôle clé au cours de l'évolution dans la transmission des signaux entre les milieux extra- et intracellulaires, les protéines à 7TM constituent aujourd'hui des cibles pharmacologiques privilégiées. Elles sont la cible d'environ 30 \% des médicaments actuellement commercialisés $[4,5]$. II convient toutefois de noter que ces médicaments ne ciblent qu'une partie des protéines à 7TM (60 à 80 membres), suggérant que la plupart de ces protéines restent inexploitées. Dans ce contexte, il est intéressant de remarquer que le ligand naturel d'une fraction non négligeable des protéines à 7TM reste toujours à identifier. Ces protéines sont appelées orphelines de ligand. Après une période de « désorphelinisation » (identification du ligand naturel) très intense et productive dans les années 1980 à 1990 , très peu de ces récepteurs orphelins sont «désorphelinisés » de nos jours $[6,7]$. 


\section{Implication des fonctions indépendantes des ligands dans la conservation des protéines orphelines à 7TM au cours de l'évolution}

Le nombre relativement élevé de protéines à 7TM orphelines de ligand (environ une centaine) et les difficultés croissantes pour leur attribuer un ligand ont conduit à considérer des hypothèses alternatives pour expliquer la signification physiologique de ces protéines et leur conservation au cours de l'évolution. L'idée a ainsi émergé qu'au moins une partie de ces protéines à 7TM orphelines de ligand étaient de vrais orphelins sans ligand naturel (bien qu'il soit difficile d'exclure l'absence de ligand naturel de façon définitive) [8]. En partant de ce constat, on ne peut plus parler de récepteur au sens strict, et l'utilisation du terme «protéines orphelines à 7TM » paraît plus adéquate. Pour comprendre le rôle de ces protéines, il a fallu chercher de nouvelles fonctions, indépendantes de la fixation d'un ligand.

Aujourd'hui, deux types de fonctions ont été identifiés : une activité constitutive de ces protéines (voir plus loin) et la modulation de la fonction d'une autre protéine à 7TM qui répond à son propre ligand, par une association au sein d'un complexe hétéromérique. Une multitude d'études montrent que les protéines à 7TM peuvent avoir une activité constitutive importante [9] et former des homomères mais également des hétéromères avec d'autres récepteurs $[10,11]$. Il est important de noter que l'hétéromérisation peut modifier les propriétés fonctionnelles des deux partenaires constituant l'hétéromère. Enfin, le phénomène d'homo- et hétéromérisation s'applique également aux protéines orphelines à 7TM [8], et plusieurs exemples ont été rapportés dans la littérature. Le récepteur $G_{B B} A_{B}$ (acide $\gamma$-aminobutyrique $B$ ), composé de deux sous-unités GBl et $G B 2$, est certainement l'exemple le mieux étudié [12]. GB2 est une protéine orpheline à 7TM qui est nécessaire à l'export de la sous-unité $G B l$ vers la surface cellulaire. Une fois à la surface, GB1 lie le GABA et GB2 se couple à la protéine Gi pour déclencher la signalisation intracellulaire. Le fonctionnement des récepteurs gustatifs repose également sur la formation d'hétéromères obligatoires entre protéines à 7TM orphelines et non orphelines. L'hétéromère entre TIR2 (taste receptor, type 1, member 2) et $T I R 3$ reconnaît des molécules sucrées, et l'hétéromère entre TlRl et TlR3 des substances amères, comme le glutamate [13, 14]. Dans ces deux cas, la sous-unité TIR3 est une protéine orpheline qui forme un hétéromère avec TlRl et TlR2 qui lient les ligands. L'hétéromère entre la protéine orpheline à 7TM GPR50 ( $G$ protein-coupled receptor 50) et le récepteur $M T_{1}$ de la mélatonine est un autre exemple intéressant [15]. GPR50 empêche le récepteur $M T_{1}$ de lier son ligand dans l'hétéromère et de se coupler à la protéine Gi ainsi qu'à la $\beta$-arrestine. Dans cet hétéromère, la longue extrémité carboxy-terminale de GPR50 joue un rôle crucial car elle empêche le recrutement de ces partenaires d'interaction, ce qui abolit de façon indirecte la liaison de haute affinité de la mélatonine.

\section{Protéines virales orphelines à 7TM et détournement des fonctions de la cellule hôte}

Les protéines virales orphelines à 7TM : 7TM-v

Très récemment, il a été rapporté dans la littérature des exemples de protéines à 7TM synthétisées par des virus (protéines à 7TM-v) de la famille des virus de l'herpès utilisant cette stratégie d'hétéromérisation, avec d'autres protéines à 7TM, pour détourner à leur avantage les propriétés de la cellule infectée. En effet, il est connu depuis longtemps que le génome de certains virus de l'herpès contient des gènes codant pour des protéines ayant une organisation prédite en 7TM et une homologie avec les récepteurs de chimiokines $[16,17]$. On pense même que ces protéines virales ont été transférées du génome des cellules hôtes vers le génome viral au cours de l'évolution. Parmi les différents virus de l'herpès, trois sont bien connus pour exprimer des protéines à 7TM-v : le virus associé au sarcome de Kaposi (KSHV), le virus Epstein-Barr (EBV) et le cytomégalovirus humain (CMVH). Tous les trois conduisent à une infection latente et persistante chez l'homme. Le KSHV et I'EBV jouent un rôle dans la cancérogenèse et expriment chacun une seule protéine à $7 T M-v$, respectivement appelée 0RF74 et BILFl. Le CMVH module les fonctions immunitaires de l'hôte et exprime quatre protéines à 7TM-v : US27, US28, UL33 et UL78. Bien que l'ensemble de ces protéines à $7 \mathrm{TM}-\mathrm{v}$ partagent une certaine homologie de séquence avec les récepteurs de chimiokines, uniquement deux, ORF74 et US28, sont connues aujourd'hui pour leur capacité à lier des chimiokines. Comme ces deux protéines à $7 \mathrm{TM}-\mathrm{v}$ peuvent lier plusieurs types de chimiokines, on constate une activation importante de la cellule hôte due à la stimulation de ces protéines virales, et ce malgré l'inactivation des récepteurs de chimiokines de la cellule hôte en raison de la séquestration des chimiokines par ces protéines virales. De façon intéressante, les autres protéines à 7TM-v sont des protéines orphelines qui ont probablement perdu leur capacité de lier les chimiokines au cours de l'évolution. La présence d'une forte activité constitutive en absence de ligand est commune aux deux types de protéines à 7TM-v (orphelines et non orphelines). Cela veut dire que la simple expression de ces protéines est suffisante pour déclencher et perturber la signalisation intracellulaire de la cellule hôte. L'impact de l'activité constitutive sur les propriétés pathogènes a surtout été montré pour ORF74, BILF1, US28 et UL33 $[16,17]$. 


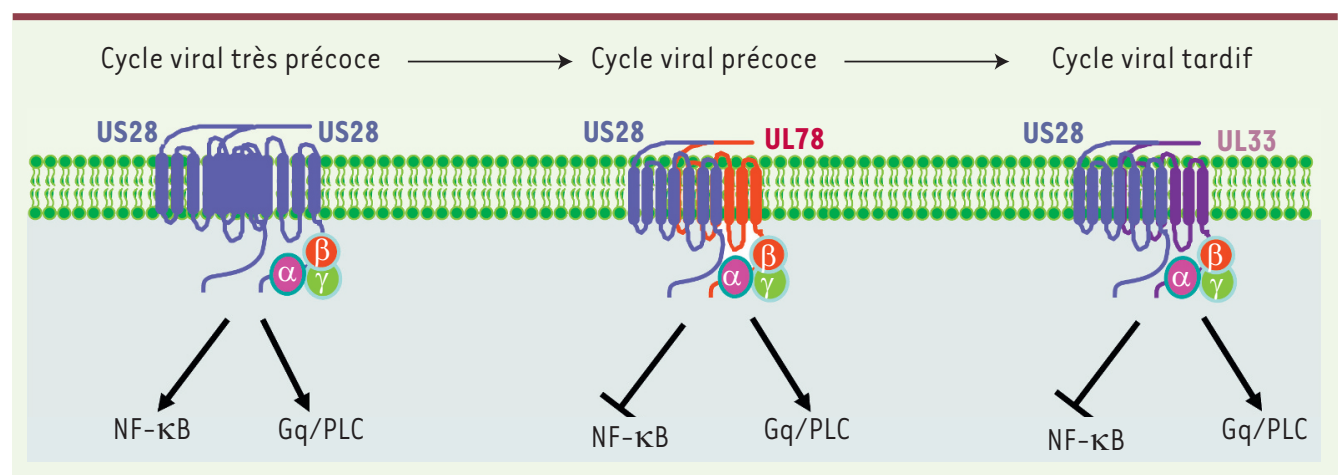

Figure 1. Formation hypothétique des hétéromères de protéines à 7TM synthétisées par le CMVH au cours du cycle viral. L'hétéromérisation de US28 avec UL33 et UL78 permettrait de moduler sélectivement la voie NF-KB constitutivement activée par l'homomère US28. Ceci constituerait un moyen de réguler la signalisation de la cellule hôte infectée par ce virus. PLC : phospholipase C.

\section{Hétéromérisation des protéines virales et humaines à 7TM}

Avec la notion de l'hétéromérisation des protéines à 7TM humaines, la question se posait de l'existence de ce même phénomène pour les protéines à 7TM-v. Une première étude in vitro en 2008 suggérait qu'une protéine à $7 \mathrm{TM}-\mathrm{v}$, comme BILFl, peut former des homomères, mais aussi des hétéromères avec des récepteurs de chimiokines humains [18]. Curieusement, BILFl semble interagir avec la plupart des récepteurs de chimiokines testés dans des cellules dans lesquelles ils ont été exprimés, comme les récepteurs CCR6, CCR7, CCR9, CCR10, CXCR3, CXCR4, CXCR5, et CXCR7 ${ }^{1}$ [27]. La réalité de ces interactions dans des cellules exprimant des récepteurs de chimiokines endogènes et infectées par l'EBV, ainsi que les conséquences fonctionnelles, restent inconnues. Une deuxième étude sur l'hétéromère BILFI/CXCR4 a permis de montrer que BILFl recrute, grâce à son activité constitutive, les protéines Gi situées dans son entourage, les séquestrant de manière à les rendre inutilisables par d'autres RCPG comme le récepteur CXCR4 [19]. Ce mécanisme montre que l'hétéromérisation des protéines à 7TM ne sert pas uniquement à modifier les propriétés des protéines impliquées dans l'hétéromère par contact direct, mais également à modifier de façon indirecte l'environnement à proximité des récepteurs (séquestration de molécules de signalisation).

Le phénomène d'homomérisation a par la suite été démontré pour M78 qui est exprimé par le cytomégalovirus murin [20] et les phénomènes d'homo- et d'hétéromérisation pour US27, US28, UL33, UL78 du CMVH $[21,22]$. Une première idée des conséquences fonctionnelles de ces associations a été révélée par l'étude de l'influence de la coexpression des protéines orphelines à 7TM-v (US27, UL33 et UL78) sur l'activité constitutive de US28. L'activation constitutive de la voie NF-kB par US28 a été complètement supprimée par la présence de UL33 et UL78, mais pas par celle de US27 (Figure 1). En revanche, aucun effet n'a

${ }^{1}$ Selon le nombre d'acides aminés présents entre les deux premières cystéines (situées dans la partie amino-terminale), les chimiokines sont classées en quatre groupes: $C C, C X C, C X 3 C$ ainsi que le groupe $C$ qui comprend la lymphotactine (XCL1), seule chimiokine contenant uniquement deux cystéines. La nomenclature des récepteurs de chimiokines est basée sur le groupe de chimiokines auquel son ligand appartient. Ainsi, les récepteurs correspondant aux chimiokines du groupe $C X C$ seront appelés $C X C R 1,2,3$, 4, etc. été observé sur la voie $\mathrm{Gq} / \mathrm{PLC}$ (phospholipase C) également activée constitutivement en présence de US28. Cela montre l'impact que peut avoir une protéine orpheline à 7TM-v sur l'activité constitutive d'une autre protéine à 7TM-v. La modulation sélective de la voie NF- $\kappa B$ par l'hétéromérisation de US28 pourrait être importante pour la latence et la réactivation du $\mathrm{CMVH}$. Comme les quatre protéines orphelines à 7TM-v du CMVH ont des cinétiques d'expression différentes au cours du cycle viral, on peut facilement supposer que cette hétéromérisation est un moyen pour le CMVH de finement contrôler la signalisation de la cellule hôte. En effet, US28 et UL78, toutes les deux coexprimées pendant les phases précoces de l'infection virale, pourraient inhiber l'activation constitutive de la voie NF-KB par US28 (Figure 1). Pendant les phases tardives de l'infection, I'activité constitutive de US28 pourrait être régulée par son hétéromérisation avec UL33. Par conséquent, on peut s'attendre à ce que l'activation constitutive de la voie NF-KB par US28 programme la cellule dans les phases très précoces de façon à ce que celle-ci soit inhibée par la suite par hétéromérisation avec UL33 et UL78. En même temps, l'effet activateur de US28 sur la voie $\mathrm{Gq} / \mathrm{PLC}$ perdurerait tout au long du cycle viral.

Impact des protéines virales orphelines à 7TM sur les récepteurs de chimiokines

\section{Modulation de CCR5 et CXCR4 par UL33 et UL78}

Des résultats récents de notre laboratoire montrent que les protéines orphelines à 7TM-v peuvent aussi avoir un impact sur la fonction des récepteurs de chimiokines exprimés par la cellule hôte [23]. En effet, nous avons montré que les protéines orphelines à 7TM-v, UL33 et UL78, modulent les fonctions de CCR5 et CXCR4, deux récepteurs de chimiokines. Ces deux récepteurs sont exprimés par plusieurs types cellulaires du système immunitaire. CXCR4 est en plus fortement exprimé dans des tissus cancéreux. Ces deux récepteurs sont connus pour réguler la migration cellulaire et la réponse inflammatoire [28] et fonctionner en tant que corécepteur du virus de l'immunodéficience humain (VIH). 


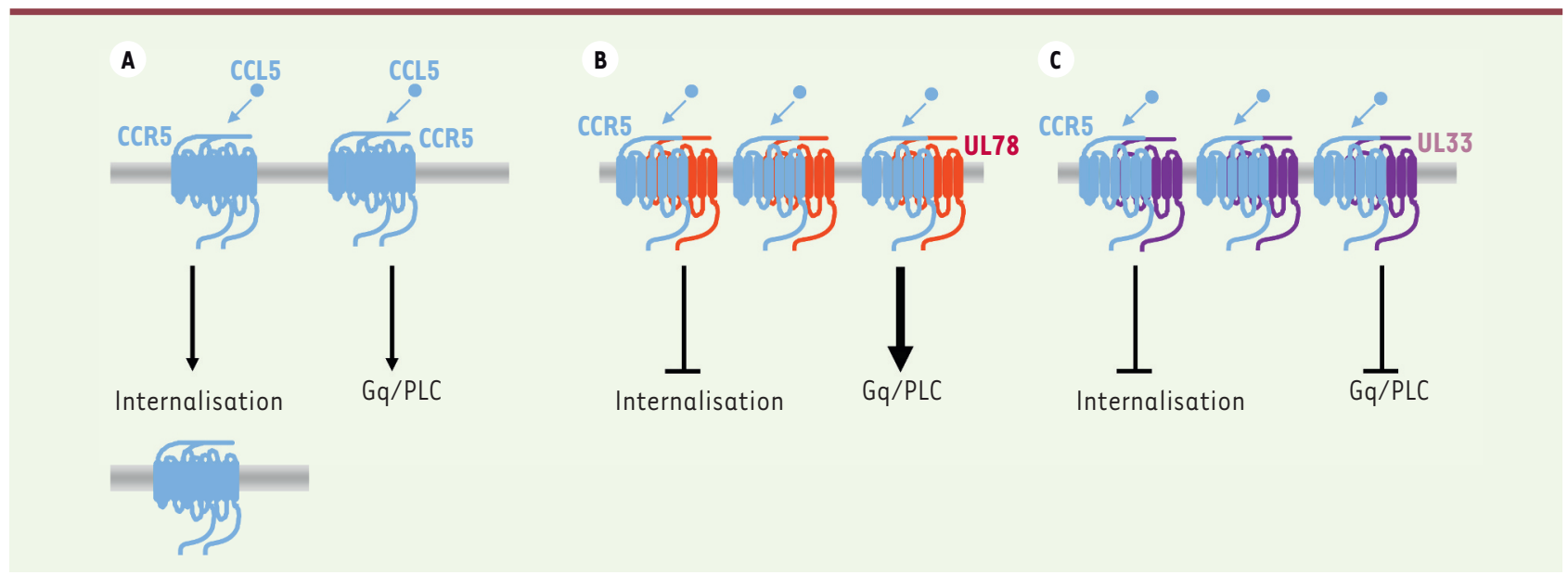

Figure 2. Effet des protéines orphelines à 7TM UL33 et UL78 sur la fonction du récepteur de chimiokine CCR5 dans les cellules THP-1. A. La liaison de l'agoniste CCL5 sur l'homomère CCR5 induit une activation de la voie Gq/PLC et l'internalisation des récepteurs CCR5. B. Associé à UL78, CCR5 n'est plus internalisé et sa signalisation est augmentée. C. À l'inverse, I'hétéromérisation de CCR5 avec UL33 abolit toutes les fonctions de CCR5. Dans les deux cas (B) et (C), l'hétéromérisation augmente l'expression de CCR5 à la surface cellulaire.

L'expression de UL33 ou UL78 dans des cellules monocytaires THP-1 (human acute monocytic leukemia cell line) exprimant CCR5 et CXCR4 de façon endogène modifie les fonctions de ces derniers à plusieurs niveaux (Figures 2, 3). CCR5 et CXCR4 sont connus pour être internalisés rapidement à la suite de leur activation respective par les chimiokines CCL5 et CXCL12. De façon intéressante, la présence de UL33 ou UL78 bloque complètement l'endocytose de CCR5. En accord avec ces observations, une augmentation de la quantité de CCR5 à la surface cellulaire est observée à l'état basal, en absence de stimulation. Dans le cas de UL78, cela conduit à une signalisation accrue de CCR5 par la voie Gq/PLC. Dans des cellules exprimant UL33, l'activation de CCR5 abolit l'activation de la voie Gq/PLC, probablement à cause de la régulation négative de cette voie par l'activité constitutive de UL33. Quant à l'endocytose de CXCR4 induite par CXCL12, elle n'est pas affectée par UL33 ou UL78 qui sont cointernalisées avec CXCR4. En revanche, ces deux protéines orphelines à $7 T M-v$ provoquent une diminution du nombre de CXCR4 à la surface cellulaire à l'état basal, ce qui pourrait expliquer la diminution de l'activation de la voie Gq/PLC par CCL5 et CCL12. Promouvoir la migration cellulaire est une fonction importante de CCR5 et CXCR4. II s'agit ici d'un phénomène qui est dépendant de la protéine Gi. L'expression de UL33 et UL78 diminue la migration des cellules THP-1 induite par CCL5 ou CXCL12.

En conclusion, UL33 et UL78 ont des effets différentiels sur l'expression de surface de CCR5 (augmentation) et CXCR4 (diminution) qui corrèlent bien avec l'amplitude de l'activation de la voie Gq/PLC par ces deux récepteurs de chimiokines. En revanche, l'effet de UL33 et UL78 sur la migration des cellules THP-1 induite par CCL5 et CXCL12 est négatif.

\section{Modification de l'interaction de cellule avec le VIH}

Comme plusieurs études suggèrent un rôle de l'oligomérisation du CCR5 et CXCR4 dans leurs propriétés de corécepteur du VIH [24], nous nous sommes intéressés à l'effet de UL33 et UL78 sur ce phénomène.
Nous avons donc infecté des cellules THP- 1 exprimant soit UL33 soit UL78 avec la souche JR-CSF (tropisme macrophagique et utilisant préférentiellement le corécepteur CCR5) ou la souche LAI (tropisme lymphoïde et utilisant préférentiellement le corécepteur (XCR4). L'infection primaire des cellules THP-1 par ces deux virus a été bien moindre dans les cellules exprimant UL33 ou UL78, ce qui suggère que des cellules précédemment infectées par le CMVH seraient partiellement protégées de la surinfection par le VIH. Les causes de cet effet bénéfique de UL33 et UL78 ne sont pas encore bien comprises. II semblerait que l'expression de surface du récepteur principal du VIH, le CD4, ainsi que son interaction avec CCR5 et CXCR4, ne soient pas modifiées par l'expression des deux protéines orphelines à 7TM-v. II est probable que UL33 et UL78 modifient des propriétés structurales de CCR5 et CXCR4 de manière à imposer, par exemple, une conformation aux deux récepteurs dans les hétéromères. Cette conformation aurait pour conséquence de diminuer l'interaction avec l'enveloppe du VIH ou de masquer des épitopes importants pour la reconnaissance par le virus. On peut espérer qu'une meilleure compréhension de cet effet inhibiteur de UL33 et UL78 sur les propriétés des corécepteurs du VIH conduise, à terme, à de nouvelles pistes thérapeutiques contre ce virus : une stratégie pourrait être le développement de petites molécules mimant l'effet inhibiteur de UL33 et UL78 sur CCR5 et CXCR4. En conclusion, ces résultats soulignent que les protéines orphelines à $7 T M-v$, comme UL33 et UL78, peuvent moduler les fonctions de la cellule hôte à plusieurs niveaux, et même modifier l'interaction de cette dernière avec des virus comme le VIH. 


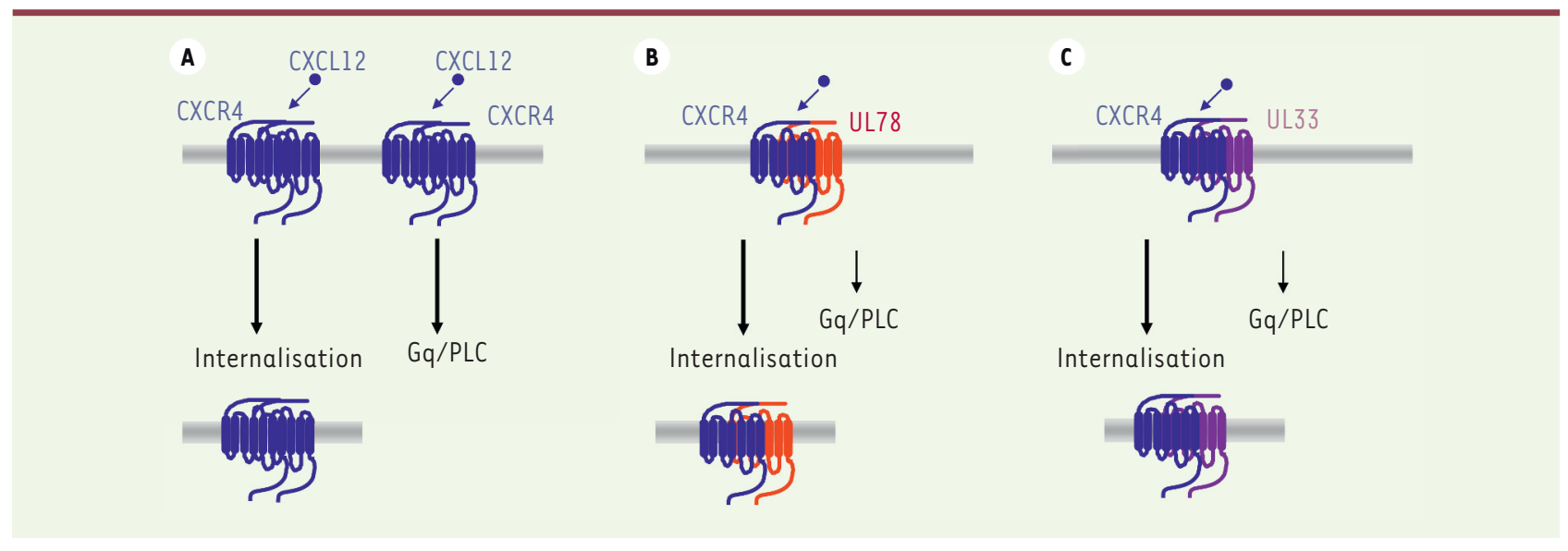

Figure 3. Régulation de la signalisation de l'homomère CXCR4 par les protéines orphelines à 7TM UL78 et UL33 dans les cellules THP-1. A. L'activation du récepteur CXCR4 par son agoniste CXCL12 induit l'activation de la voie Gq/PLC et l'internalisation de CXCR4. B. L’hétéromérisation de UL78 avec CXCR4 induit la cointernalisation UL78/CXCR4 et une diminution de la voie Gq/PLC après liaison de l'agoniste. C. Les mêmes effets sont observés pour l'hétéromère CXCR4/UL33.

\section{Conclusion}

Une part importante des protéines à 7TM résiste à leur « désorphelinisation ». Cette situation a suscité des hypothèses alternatives qui ne reposent pas sur leur propriété de lier un ligand, mais par exemple sur la modulation de la fonction des protéines à 7TM fixant un ligand, dans des complexes hétéromériques. Des données récentes, présentées dans cet article, suggèrent que certains virus appartenant à la famille des virus de l'herpès utilisent cette même stratégie pour moduler l'effet de leurs propres protéines à 7TM sur la cellule hôte (exemple des hétéromères UL33/US28 et UL78/US28) ou pour moduler directement la fonction des protéines à 7TM de la cellule dans des hétéromères communs (BILFl/CXCR4, UL33 ou UL78/CCR5 ou CXCR4). Les virus sont soumis à une pression évolutive importante et il est intéressant de se demander pourquoi les protéines orphelines à 7TM-v n'ont pas été éliminées du génome viral malgré leur perte de la capacité à lier un ligand? L'explication la plus simple est qu'il existe d'autres fonctions essentielles à la propagation et ou la survie du virus qui ne nécessitent pas l'intervention d'un ligand. L'activité constitutive est certainement une de ces propriétés essentielles, et I'hétéromérisation avec d'autres protéines à 7TM peut en être une autre. Les virus ont toujours été une très grande source d'inspiration pour mieux comprendre le fonctionnement de leurs cellules hôtes. Ils pourraient, cette fois encore, nous être utiles pour mieux comprendre l'importance de la formation des hétéromères pour le fonctionnement des protéines orphelines à 7TM et, plus généralement, le phénomène d'hétéromérisation des protéines à 7TM. $\diamond$

\section{SUMMARY}

Oligomerization of human and viral 7TM proteins: a new viral strategy to manipulate host cells

$G$ protein-coupled receptors (GPCR), also called seven transmembrane domain (7TM) proteins, represent the largest family of membrane receptors with approximately 900 members in humans. Although a substantial number of 7TM proteins have been matched with endogenous ligands, for many of them no ligand has been identified raising questions about their function. Ligand-independent functions have been proposed for several of these so-called orphan 7TM proteins such as the modulation of the function of 7TM proteins with identified ligand through the formation of heteromeric complexes. Interestingly, viruses are using a similar strategy to hijack the host cell signaling machinery and to promote virus replication and dissemination. Indeed, to affect host cell function, several viruses encode orphan 7TM proteins that heteromerize either with other virally-encoded or with host-encoded 7TM proteins with identified ligands. This highlights the strategic importance of 7TM protein signaling and heteromerization for the regulation of cellular homeostasis. $\diamond$

\section{LIENS D’INTÉRÊT}

Les auteurs déclarent n'avoir aucun lien d'intérêt concernant les données publiées dans cet article.

\section{REMERCIEMENTS}

Nous tenons à remercier l'Association pour la recherche sur le cancer (ARC, $n^{\circ}$ 5051), l'Institut national de la santé et de la recherche médicale (Inserm), le Centre national de la recherche scientifique (CNRS), la Fondation pour la recherche médicale (FRM) et l'université Paris Descartes (bourse postdoctorale pour Kenjiro Tadagaki) pour leurs soutiens financiers, ainsi que Jean-Luc Guillaume (Institut Cochin, Paris) pour ses commentaires sur le manuscrit. 


\section{RéFÉRENCES}

1. Almen MS, Nordstrom KJ, Fredriksson R, Schioth HB. Mapping the human membrane proteome: a majority of the human membrane proteins can be classified according to function and evolutionary origin. BMC Biol $2009 ; 7: 50$.

2. Rosenbaum DM, Rasmussen SG, Kobilka BK. The structure and function of G-protein-coupled receptors. Nature $2009 ; 459$ : 356-63

3. Nordstrom KJ, Sallman Almen M, Edstam MM, et al. Independent HH search, Needleman-Wunschbased, and motif analyses reveal the overall hierarchy for most of the $\mathrm{G}$ protein-coupled receptor families. Mol Biol Evol $2011 ; 28: 2471-80$

4. Gruber CW, Muttenthaler M, Freissmuth M. Ligand-based peptide design and combinatorial peptide libraries to target $G$ protein-coupled receptors. Curr Pharm Des $2010 ; 16: 3071-88$.

5. Rask-Andersen M, Almen MS, Schioth HB. Trends in the exploitation of novel drug targets. Nat Rev Drug Discov $2011 ; 10: 579-90$.

6. Levoye $A$, Jockers $R$. L'hétérodimérisation des récepteurs couplés aux protéines $G$ : une nouvelle voie vers la découverte de fonctions pour les récepteurs orphelins? Med Sci (Paris) 2007 ; 23 : 746-50.

7. Levoye A, Jockers R. Alternative drug discovery approaches for orphan GPCRs. Drug Discov Today $2008 ; 13: 52-8$

8. Levoye A, Dam J, Ayoub MA, et al. Do orphan G-protein-coupled receptors have ligandindependent functions? New insights from receptor heterodimers. EMBO Rep 2006 ; 7 : 1094-8.

9. Arrang JM, Morisset $\mathrm{S}$, Gbahou F. Constitutive activity of the histamine $\mathrm{H} 3$ receptor. Trends Pharmacol Sci $2007 ; 28: 350-7$.

10. Milligan G. G protein-coupled receptor hetero-dimerization: contribution to pharmacology and function. BrJ Pharmacol $2009 ; 158: 5-14$

11. Tadagaki K, Jockers R, Kamal M. History and biological significance of GPCR heteromerization in the neuroendocrine system. Neuroendocrinology $2012 ; 95: 223-31$.

12. Pin JP, Kniazeff J, Liu J, et al. Allosteric functioning of dimeric class C G-protein-coupled receptors. FEBS J $2005 ; 272: 2947-55$.

13. Nelson G, Hoon MA, Chandrashekar J, et al. Mammalian sweet taste receptors. Cell 2001 ; 106 : 381-90.

14. Nelson G, Chandrashekar J, Hoon MA, et al. An amino-acid taste receptor. Nature 2002 ; 416 : 199-202

15. Levoye A, Dam J, Ayoub MA, et al. The orphan GPR50 receptor specifically inhibits MT(1) melatonin receptor function through heterodimerization. $E M B O J 2006 ; 25: 3012-23$.

16. Slinger $\varepsilon$, Langemeijer $\varepsilon$, Siderius $M$, et al. Herpesvirus-encoded GPCRs rewire cellular signaling. Mol Cell Endocrinol $2011 ; 331: 179-84$

17. Rosenkilde MM, Smit MJ, Waldhoer M. Structure, function and physiological consequences of virally encoded chemokine seven transmembrane receptors. Br J Pharmacol 2008 ; 153 : S154-66.
18. Vischer HF, Nijmeijer S, Smit MJ, Leurs R. Viral hijacking of human receptors through heterodimerization. Biochem Biophys Res Commun 2008 ; 377 93-7.

19. Nijmeijer S, Leurs R, Smit MJ, Vischer HF. The Epstein-Barr virus-encoded $G$ protein-coupled receptor BILFI hetero-oligomerizes with human CXCR4 scavenges Galphai proteins, and constitutively impairs CXCR4 functioning. ) Biol Chem $2010 ; 285: 29632-41$.

20. Sharp EL, Davis-Poynter NJ, Farrell HE. Analysis of the subcellular trafficking properties of murine cytomegalovirus M78, a 7 transmembrane receptor homologue.J Gen Virol 2009 ; 90 : 59-68.

21. Tschische P, Tadagaki K, Kamal M, et al. Heteromerization of human cytomegalovirus encoded chemokine receptors. Biochem Pharmacol 2011 ; $82: 610-9$

22. Wagner S, Arnold F, Wu Z, et al. The 7-transmembrane protein homologue UL78 of the human cytomegalovirus forms homomers and traffics between the plasma membrane and different intracellular compartments. Arch Virol $2012 ; 157: 935-49$.

23. Tadagaki K, Tudor D, Gbahou F, et al. Human cytomegalovirus-encoded UL33 and UL78 heteromerize with host CCR5 and CXCR4 impairing their HIV coreceptor activity. Blood $2012 ; 119: 4908-18$.

24. Springael Jy, Urizar $\varepsilon$, Parmentier M. Dimerization of chemokine receptor and its functional consequences. Cytokine Growth Factor Rev $2005 ; 16$ : 611-23.

25. Lebon $\mathrm{G}$, Tate $\mathrm{CG}$. Les récepteurs couplés aux protéines $\mathrm{G}$ dans la lumière. Med Sci (Paris) $2012 ; 28: 876-82$

26. Granier S. Structure des récepteurs mu et delta des opiacés. Med Sci (Paris) $2012 ; 28: 870-5$

27. Combadière $B$, Combadière $C$, Deterre $P$. Les chimiokines : un réseau sophistiqué de guidage cellulaire. Med Sci (Paris) 2007 ; 23 :173-9.

28. Desjardins SF, Berchiche YA, Haddad $\varepsilon$, Heveker N. CXCR4, un récepteur de chimiokine aux multiples talents. Med Sci (Paris) $2007 ; 23: 980-4$.

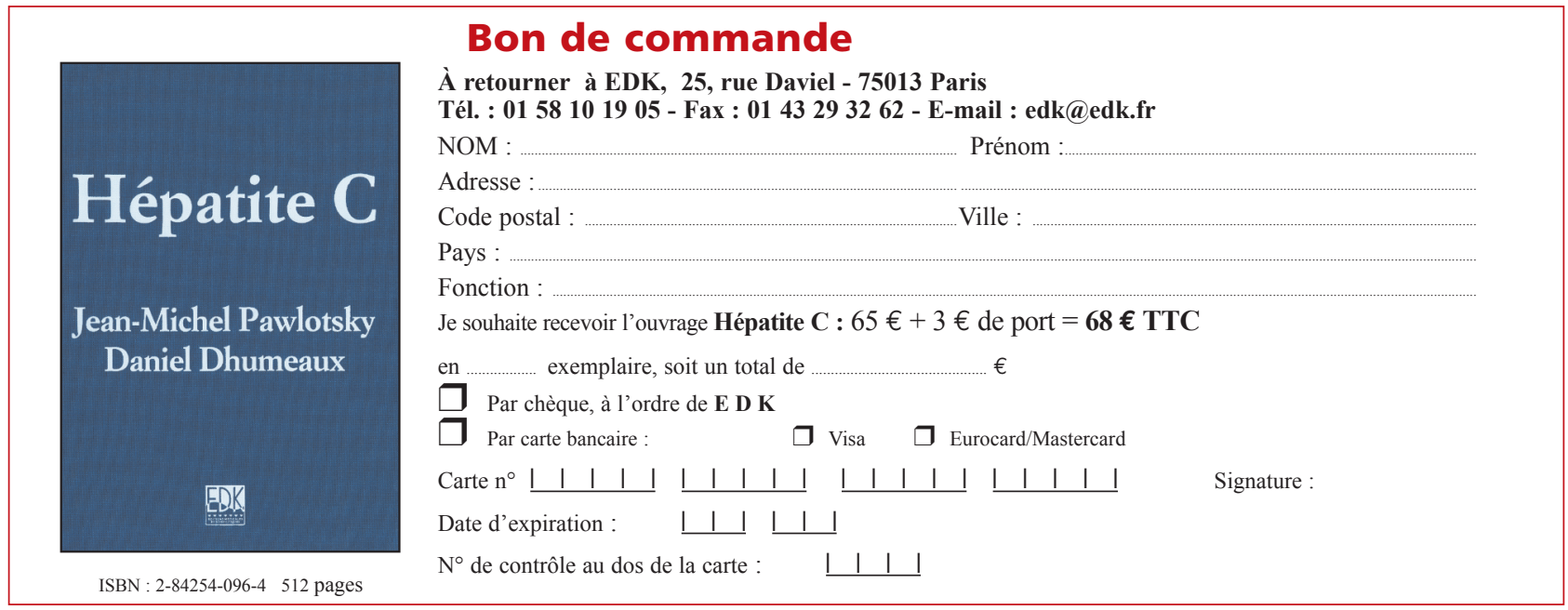

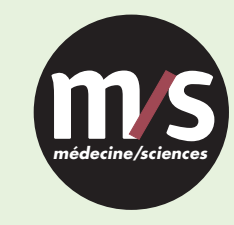

Tarifs d'abonnement $\mathrm{m} / \mathrm{s}-2012$

Abonnez-vous à médecine/sciences
$>$ Grâce à $m / s$, vivez en direct les progrès des sciences biologiques et médicales

Bulletin d'abonnement page 804 dans ce numéro de $\mathrm{m} / \mathrm{s}$
TIRÉS À PART

R. Jockers 